\title{
Emotion dysregulation in adults suffering from attention deficit hyperactivity disorder (ADHD), a comparison with borderline personality disorder (BPD)
}

Eva Rüfenacht ${ }^{1 *}$, Sebastian Euler ${ }^{2}$, Paco Prada ${ }^{1}$, Rosetta Nicastro ${ }^{1}$, Karen Dieben ${ }^{1}$, Roland Hasler ${ }^{1}$, Eléonore Pham Nader Perroud ${ }^{1,3+}$ and Sébastien Weibel ${ }^{4,5,6+}$

\begin{abstract}
Background: Emotional dysregulation (ED) is now considered as an important symptom of attention deficit hyperactivity disorder (ADHD). It is believed to have a considerable impact on the severity of the disorder, one's global functioning, and the prognosis. Our research aimed to evaluate and compare ED and cognitive emotional regulation strategies between ADHD and borderline personality disorder (BPD) patients.

Methods: Four hundred six French-speaking outpatients ( $N=279$ ADHD, N=70 BPD, N=60 BPD + ADHD) were assessed with the Emotion Reactivity Scale (ERS), the Cognitive Emotional Regulation Questionnaire (CERQ), The Basic Empathy Scale (BES-A), the Adult ADHD Self-Report Scale (ARSV-V1.1) and the Beck Depression Inventory II (BDI-II). ADHD, BPD and comorbid patients were compared with each other and with samples of controls extracted from already published data.

Results: ADHD patients, although having higher ED than samples derived from the general population, had less ED, better control over their emotions with higher use of adaptive cognitive strategies and lesser use of non-adaptive strategies than BPD patients. However, ADHD subjects had similar scores as BPD subjects when looking at difficulties in perceiving self and others. ED generated considerable distress in all groups and was also positively associated with ADHD symptomatology. ADHD patients with comorbid BPD had the highest scores of ED.

Conclusions: Our results suggest that there may be similarly inefficient cognitive emotional regulation skills leading to ED in both disorders (ADHD and BPD). However, ADHD patients showed a higher use of adaptive cognitive emotional strategies and a lower level of ED than BPD patients.

Keywords: Emotional dysregulation, Attention deficit hyperactivity disorder, Borderline personality disorder, Cognitive emotion regulation strategies, Comorbid ADHD and borderline personality disorder, Metacognitive abilities, Emotional perception
\end{abstract}

\section{Background}

There is increasing evidence of a close link between emotional symptoms and attention deficit hyperactivity disorder (ADHD) [1-3]. Several studies suggest that besides attentional and hyperactive-impulsive symptoms,

\footnotetext{
*Correspondence: evarufenacht@gmail.com

${ }^{\dagger}$ Nader Perroud and Sébastien Weibel contributed equally to this work.

${ }^{1}$ TRE Unit, Department of Psychiatry, Division of Psychiatric Specialties, Department of Mental Health and Psychiatry, University Hospitals of Geneva, 20bis rue de Lausanne, Geneva, Switzerland

Full list of author information is available at the end of the article
}

emotional dysregulation (ED) is a core symptom of ADHD, both in childhood and adulthood [4-6].

Studies have estimated that 34 to $70 \%$ of adults suffering from ADHD have difficulties regulating their emotions [4-6]. These emotional symptoms have been associated with poor global functioning including lower quality of life, interpersonal and professional difficulties, poor prognosis, and severity of attentional and hyperactive-impulsive symptoms in adults as well as in children [1, 4, 7-9]. Research has shown that ED in ADHD is similar to the nature of ED found in borderline

(c) The Author(s). 2019 Open Access This article is distributed under the terms of the Creative Commons Attribution 4.0 International License (http://creativecommons.org/licenses/by/4.0/), which permits unrestricted use, distribution, and 
personality disorder (BPD), comprising of increased instability and intensity of negative emotions and a slow return to emotional baseline when activated $[4,10]$. ED in BPD is considered a fundamental mechanism of the disorder, and this dimension concerns at least two of the DSM- 5 criteria for the disorder. This makes BPD the "gold standard" disorder to which other disorders (such as ADHD) can be compared to assess their level of ED. The frequent comorbidity between ADHD and BPD (around one third of BPD patients have ADHD, and vice versa) suggests common mechanisms and aetiology [11, 12]. There is some evidence that ED symptoms mediate the relationship between retrospectively assessed childhood ADHD and adulthood BPD symptoms [13]. Additionally, ADHD has been identified as a potential risk factor for adulthood BPD development [14]. Yet few studies directly compared ED in BPD and ADHD and none considered comorbid ADHD+BPD patients [14-16].

Besides the usual description of ED in BPD, namely heightened emotional reactivity to environmental stimuli, inadequate cognitive emotion regulation strategies resulting form difficulty identifying, differentiating, and labeling emotions have also been suggested as important components of ED [17-22]. BPD patients indeed engage in more maladaptive cognitive emotion regulation strategies such as 'thought suppression' (efforts to suppress emotional response to a subjective experience) and 'rumination' (disproportionate focus on emotional experiences) and show fewer adaptive strategies such as 'cognitive reappraisal' (have a different perspective on a situation to change oneself feeling) or 'acceptance' (endorse a nonjudgmental stance toward internal experiences) compared to healthy individuals [18-22]. The use of more maladaptive cognitive strategies has been associated, in BPD, with higher severity of the disorder (more harmful behaviours) [18]. Finally, poor mentalizing capacities (the process sustaining our understanding of human action as driven by mental states including, among other dimensions, empathy) are also considered to underlie ED in BPD. Indeed, poor reflective functioning, the operationalized measure of mentalizing capacity, has been directly linked to poor emotion regulation in BPD [14].

Although several hypotheses explaining ED in ADHD have been suggested, including executive function deficits, social cognitions deficit, impaired emotional perception/processing, lack of empathy and impairment in first and second order theory of mind and poor mentalizing capacities [14, 23-31], less is known about the efficiency of cognitive emotion regulation strategies used by ADHD patients to cope with emotional outbursts. Some studies on adults suffering from ADHD have associated ED and poor emotional regulation strategies such as less use of 'cognitive reappraisal' and more use of 'suppression' [32]. Poor cognitive emotion regulation skills in
ADHD have also been linked to impaired metacognitive abilities, including difficulties to focus oneself, and a diminished ability to re-evaluate goals and behaviours, prompting poor situation evaluations, thereby magnifying the associated emotional state [3, 4, 24, 32]. A better understanding of $\mathrm{ED}$, cognitive emotion regulation strategies and their correlation with ADHD disorder severity is clearly needed.

The aim of our study was therefore to compare emotional reactivity, cognitive emotional regulation strategies, and empathy in subjects suffering of ADHD to subjects with BPD, or comorbid ADHD and BPD. We also evaluated the relationship between ADHD symptoms and ED severity.

\section{Methods}

\section{Participants and procedure}

Four hundred and six French-speaking outpatients $(N=$ 279 ADHD, $N=70 \mathrm{BPD}$ and $N=60 \mathrm{BPD}+\mathrm{ADHD})$ were recruited in a specialized centre for diagnosis and treatment of adults suffering from ADHD and BPD at the University Hospitals of Geneva.

Patients underwent a clinical evaluation at their entry of the program conducted by trained psychiatrists to ascertain the diagnosis of BPD and/or ADHD according to DSM-5 criteria, and to exclude any organic condition and/or comorbid disorders that could better explain the symptoms. With the exception of ADHD and BPD (see below), other comorbidities were assessed clinically and using medical records only, and no structured interviews were used. In addition, subjects were administered the Adult ADHD Self-Report Scale-Version 1.1 part $A$ and B (ASRS v1.1) [33]; and the Borderline Symptoms CheckList (BSL-23) for BPD patients as previously described [34]. Finally, ADHD diagnosis was confirmed by the structured Diagnostic Interview for ADHD in adults (DIVA 2.0), assessing DSM-IV ADHD criteria [35] (but DSM-5 criteria were applied). BPD diagnosis was confirmed by the structured diagnostic Screening Interview for Axis II disorders (SCID-II), assessing DSM-IV BPD criteria [36].

Of note, most of the ADHD patients were free of ADHD medication when participating to the current study. Indeed, most of them were not diagnosed previously and underwent the clinical and structured evaluations (including the self-report questionnaires described below) when psychostimulants were not yet started.

All participants completed the Beck Depression Inventory II (BDI-II) to assess the current level of depression as an indirect tool to evaluate the current distress associated with suffering from ADHD or BPD [37]. BDI-II has been shown to be a good proxy to assess subjective distress associated with ADHD [32, 38]. 
The study was approved by the ethics committee of University Hospitals of Geneva and all subjects provided informed written consent.

\section{Assessment instruments used in the study}

Several instruments were used to assess emotion regulation and reactivity, cognitive strategies to avoid worries, and empathy.

The Emotion Reactivity Scale (ERS) is a self-report questionnaire enquiring about emotional experience on regular basis. It consists of 21 items measuring emotion reactivity, based on three aspects: emotion sensitivity, intensity, and persistence. Each item is rated on a 5-point Likert scale from 0 (not at all like me) to 4 (completely like me), with scores ranging from 0 to 40 for emotion sensitivity, 0 to 28 for emotion intensity and 0 to 16 for persistence, and total scores ranging from 0 to 84 [39]. The validated French version was used [40]. Studies found that total scale and subscales had good internal consistency, and factor analyses revealed that both a single-factor and a three-factor model fit the data well $[40,41]$. The scale can therefore be used with total scores and subscores.

The Cognitive Emotional Regulation Questionnaire (CERQ) is a 36-item questionnaire consisting of 9 conceptually different subscales based on different cognitive emotion regulation strategies, overall divided in two main domains: the adaptive strategies domain entails putting into perspective, positive refocusing, positive reappraisal, acceptance, refocus on planning; the non-adaptive strategies domain comprises self-blame, other-blame, rumination, and catastrophizing. Each subscale contains 4 items referring to thoughts after the experience of a threatening or stressful life event. The items are measured on a 5-point Likert scale, ranging from 1 (almost never) to 5 (almost always) [42]. The validated French version was used [43]. Exploratory and confirmatory factor analyses showed that a nine-factor model explained the data in the original and the French version $[42,43]$.

The Basic Empathy Scale in Adults (BES-A) is a 20item self-report scale, focusing on two components of empathy, the cognitive and affective components [44, 45]. Each item is rated on a 5-point Likert scale $(1=$ Strongly Disagree to $5=$ Strongly Agree; 7 reversed items), with a score ranging from 20 (deficit in empathy) to 100 (high level of empathy). Nine items evaluate cognitive empathy and 11 affective empathy. The validated French version was used [46]. The scale, initially validated in adolescents, was subsequently validated in an adult sample [45] showing that the two-factor model was appropriate.

\section{Sample of controls}

ADHD patients were compared to a sample of controls extracted from published data that have used the ERS, the CERQ or the BES-A [18, 39, 40, 45, 47, 48]. Of note, controls were not matched for age or gender.

\section{Statistics}

All analyses were performed using Stata v14. Univariate comparisons between clinical groups were conducted using the chi square test for qualitative variables (gender, comorbidity, treatment) or Fisher's exact test when assumption of frequencies for chi square test were not met, and a one-way ANOVA for quantitative variables (age, clinical scales). T-tests were used to compare ADHD patients to a sample of controls extracted from published data that have used the ERS, the CERQ or the BES-A [18, 39, 40, 45, 47, 48]. Statistical significance was accepted for $p<0.05$. Post-hoc tests were performed using pairwise comparisons (Bonferroni correction).

Questionnaire scores were analyzed with linear regression models, using the diagnostic group as a fixed variable. Models were adjusted for age and gender in the event of significant differences between groups. For all continuous predictors, we examined the assumption of normality of the distribution of residuals with residual plots. These revealed no breach of model assumptions.

Secondary analyses were performed to assess the relation between symptomatology and questionnaire scores. ASRS total score (for ADHD and ADHD+BPD groups) or BDI-II scores were added into the model as continuous predictors. Finally, association with ADHD presentation and current depressive episode were also assessed.

\section{Results}

\section{Demographic and clinical characteristics}

ADHD patients were older than the other groups $(F=6.5$; $\mathrm{df}=2 / 405 ; p=0.002)$. BPD and BPD + ADHD patients were more likely to be female than ADHD patients (X2 = 89.7; $p<0.001$ ). ADHD patients were more likely to have a job than the two other groups $(\mathrm{X} 2=9.21 ; p=0.01)$ and to have 2 or more children $(\mathrm{X} 2=10.11 ; p=0.04)$ (Table 1$)$.

Patients with ADHD were less likely to have other comorbid disorders than BPD and ADHD+BPD patients. Taken together $63.44 \%(N=177 / 279)$ of ADHD patients did have at least one comorbidity compared to $97.14 \%$ $(N=68 / 70)$ of BPD patients and $93.33 \%(N=56 / 60)$ of the ADHD+BPD patients. The use of medication differed among participants. A small fraction of patients took stimulants: $12.90 \%$ of ADHD patients, $11.66 \%$ of ADHD+BPD patients, and $1.43 \%$ of BPD patients. No patients took non-stimulant ADHD medication. The use of other medications (antipsychotics, antidepressants, benzodiazepines, mood stabilizers) were lower in ADHD patients compared to the two other groups (Table 1). The groups also differed in terms of symptomatology. ADHD symptoms as measured by the ASRS v1.1 were the highest in the BPD + ADHD group, and the lowest 
Table 1 Clinical and demographic characteristics of ADHD, BPD and comorbid ADHD+BPD patients

\begin{tabular}{|c|c|c|c|c|c|c|c|}
\hline & \multicolumn{2}{|l|}{$\begin{array}{l}\text { ADHD } \\
N=279\end{array}$} & \multicolumn{2}{|l|}{$\begin{array}{l}B P D \\
N=70\end{array}$} & \multicolumn{2}{|l|}{$\begin{array}{l}\mathrm{ADHD}+\mathrm{BPD} \\
N=60\end{array}$} & \multirow[t]{2}{*}{$P$} \\
\hline & mean or $\mathrm{N}$ & sd or $\%$ & mean or $\mathrm{N}$ & sd or $\%$ & mean or $N$ & sd or $\%$ & \\
\hline Age & 35.49 & 12.86 & 31.66 & 9.08 & 30.29 & 8.64 & 0.002 \\
\hline \multicolumn{8}{|l|}{ Gender } \\
\hline Female & 122 & 43.73 & 66 & 94.29 & 55 & 91.67 & $<0.001$ \\
\hline \multicolumn{8}{|l|}{ Civil status $^{\mathrm{a}}$} \\
\hline Single (vs not single) & 130 & 49.24 & 36 & 51.43 & 33 & 57.89 & 0.493 \\
\hline \multicolumn{8}{|l|}{ Children $^{a}$} \\
\hline 0 & 163 & 61.74 & 46 & 65.71 & 43 & 74.14 & 0.04 \\
\hline 1 & 28 & 10.61 & 13 & 18.57 & 7 & 12.07 & \\
\hline$\geq 2$ & 73 & 27.65 & 11 & 15.71 & 8 & 13.79 & \\
\hline \multicolumn{8}{|l|}{$J o b^{a}$} \\
\hline Yes (vs no) & 157 & 61.33 & 30 & 42.86 & 26 & 48.15 & 0.01 \\
\hline Years of education ${ }^{a}$ & 15.52 & 2.86 & 15.23 & 3.18 & 14.78 & 2.69 & 0.236 \\
\hline \multicolumn{8}{|l|}{ Lifetime comorbidities } \\
\hline Major depressive disorder & 122 & 43.73 & 58 & 82.86 & 39 & 65.00 & $<0.001$ \\
\hline Bipolar disorder & 6 & 2.15 & 7 & 10 & 6 & 10.00 & 0.002 \\
\hline Anxiety disorder ${ }^{b}$ & 57 & 20.43 & 58 & 82.86 & 33 & 55.00 & $<0.001$ \\
\hline Eating Disorders & 10 & 3.58 & 14 & 20 & 9 & 15.00 & $<0.001$ \\
\hline Substance use disorder & 70 & 25.09 & 24 & 34.29 & 24 & 40.00 & 0.03 \\
\hline \multicolumn{8}{|l|}{ ADHD type } \\
\hline Attentional & 109 & 39.07 & - & - & 27 & 46.55 & 0.508 \\
\hline Hyperactive-impulsive & 12 & 4.30 & - & - & 3 & 5.17 & \\
\hline Mixte & 158 & 56.63 & - & - & 28 & 48.28 & \\
\hline \multicolumn{8}{|l|}{ Medication } \\
\hline Psychostimulant & 36 & 12.90 & 1 & 1.43 & 7 & 11.66 & 0.02 \\
\hline Antidepressant & 57 & 20.43 & 36 & 51.43 & 24 & 40.00 & $<0.001$ \\
\hline Antipsychotic & 12 & 4.30 & 11 & 15.71 & 8 & 13.33 & 0.001 \\
\hline Benzodiazepine & 28 & 10.03 & 20 & 28.57 & 10 & 16.66 & $<0.001$ \\
\hline Mood stabilizer & 2 & 0.72 & 7 & 10.00 & 4 & 6.66 & $<0.001$ \\
\hline \multicolumn{8}{|l|}{ ASRS v1.1 } \\
\hline Inat. & 25.23 & 5.37 & 22.33 & 6.38 & 25.65 & 5.56 & $<0.001$ \\
\hline Hyper. & 21.23 & 6.87 & 19.92 & 6.49 & 23.88 & 7.52 & $<0.001$ \\
\hline Total & 46.47 & 10.18 & 42.25 & 11.31 & 49.53 & 10.87 & $<0.001$ \\
\hline BDI-II & 20.79 & 11.99 & 34.75 & 11.65 & 33.19 & 9.78 & $<0.001$ \\
\hline
\end{tabular}

Means, absolute numbers (N), SD, \% and comparisons ( $p$-value) between ADHD, BPD, and comorbid ADHD+BPD groups for clinical and demographic characteristics

a missing values encountered, ${ }^{b}$ Including: generalized anxiety disorder, panic disorder, social phobia and obsessive-compulsive disorder

in $\operatorname{BPD}(F=7.87 ; \mathrm{df}=2 / 403 ; p<0.001)$. BDI-II scores were higher in BPD and BPD + ADHD than in ADHD group $(F=55.79 ; \mathrm{df}=2 / 398 ; p<0.001)$ (Table 1$)$.

\section{Emotional reactivity scale}

ERS total and subscale scores are displayed in Table 2. $\mathrm{BPD}$ and $\mathrm{BPD}+\mathrm{ADHD}$ patients scored higher than ADHD patients in ERS total and in each subscale: sensibility, intensity and persistence (each $p<0.001$ ) (Table 2). BPD and BPD + ADHD did not differ.

Our ADHD patients displayed higher ED as measured by the ERS total score compared to a patient population recruited from community and local psychiatric clinics $(\mathrm{t}=4.79 ; p<0.001$; Mean: 36.66 standard deviation (SD): 17.52 vs Mean: 47.38 SD: 18.39) [39] as well as compared to French-speaking participants from the 
Table 2 Comparisons of ERS, CERQ and BES results between ADHD, BPD and comorbid ADHD+BPD

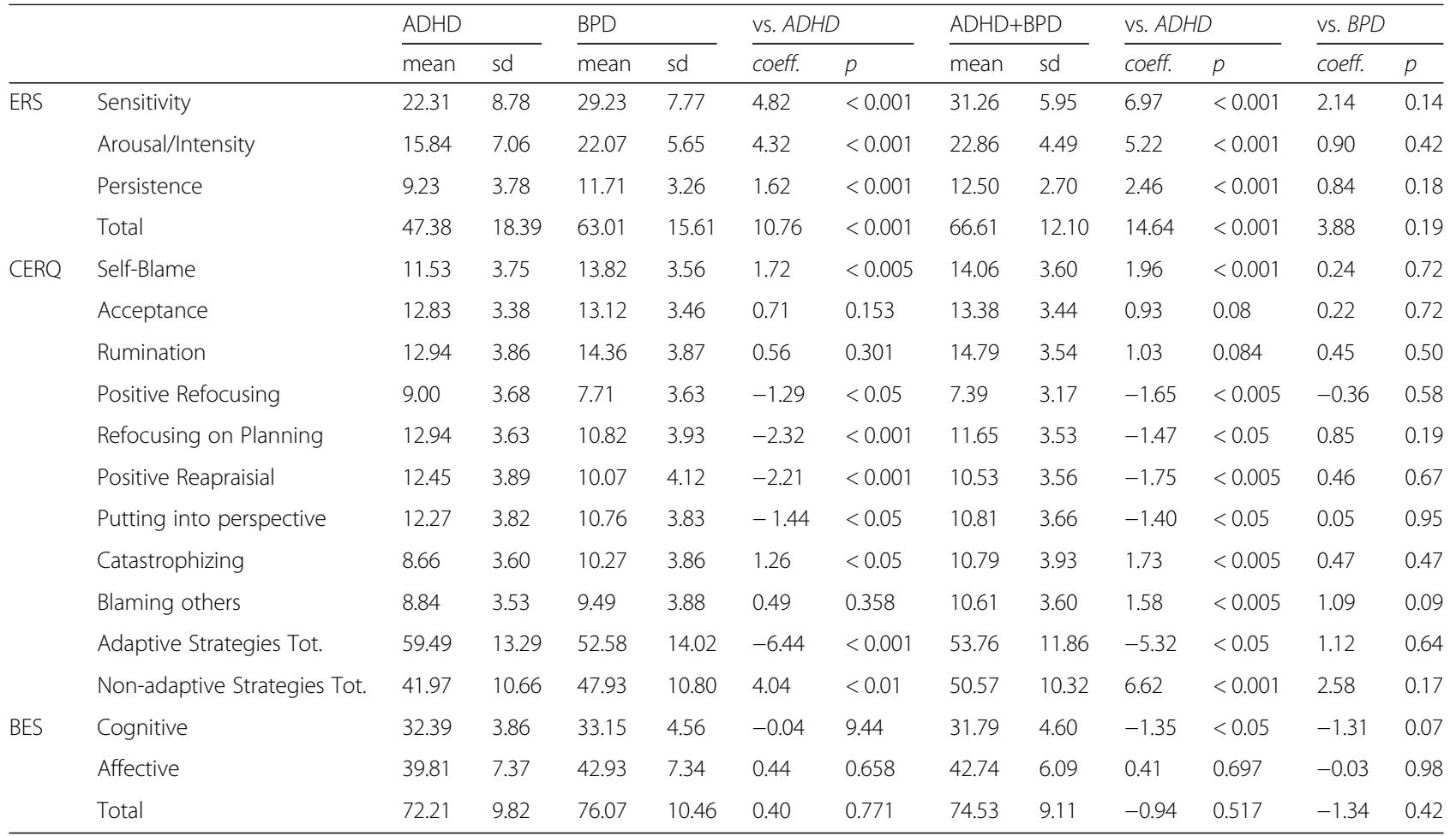

Comparisons adjusted on age and gender

Means, SD and comparisons (coefficient and $p$ value) between ADHD, BPD and comorbid ADHD+BPD for Emotion Regulation Scale (ERS), Cognitive Emotion Regulation Questionnaire (CERQ) and Basic Empathy Scale (BES)

community $(\mathrm{t}=8.03 ; p<0.001$; Mean: $35.02 \mathrm{SD}: 17.14)$ [40] (Table 3).

\section{Cognitive emotion regulation questionnaire}

ADHD patients showed higher scores for adaptive cognitive emotion regulation strategies $(\beta=-6.44 ; p<$ 0.001 and $\beta=-5.32 ; p<0.05)$, and lower scores for non-adaptive strategies $(\beta=4.04 ; p<0.01$ and $\beta=6.62$; $p<0.001)$ than BPD and ADHD+BPD patients respectively. BPD and BPD + ADHD groups did not differ. The subscale scores showed an overall similar pattern, with exception of the 'acceptance' (adaptive) and 'rumination' (non-adaptive) subscales where all three groups showed comparable scores. ADHD patients had similar scores as BPD patients in the 'blaming others' subscale but were still significantly lower than BPD + ADHD patients (Table 2).

Our ADHD patients showed poorer cognitive emotions regulation strategies as indicated by higher levels of all non-adaptive cognitive strategies than 611 adults from the general population control group $(p<$ 0.0001 for all non-adaptive strategies, $t$-values ranging from 9.12 to 14.19) [47]. Results pertaining to the adaptive cognitive strategy 'acceptance' were less consistent across samples, with overall similar results as controls (Table 3).

\section{Basic empathy scale}

The three groups did not differ on total or on cognitive or affective subscale scores, with the exception of lower cognitive empathy in BPD + ADHD than in ADHD patients $(\beta=-1.35 ; p<0.05)$ (Table 2).

Compared to a community sample of adolescents [45, 48] (who are supposed to have lower empathy than adults), our ADHD patients had significantly lower total and cognitive empathy (72.21 ( $\mathrm{SD}=9.82)$ vs. 75.11 ( $\mathrm{SD}=$ 10.20) $\mathrm{t}=-2.22 ; p=0.03$ and $32.39(\mathrm{SD}=3.86)$ vs 37.62 $(\mathrm{SD}=3.46) \mathrm{t}=-10.52 ; p<0.001$ respectively) but higher affective empathy (39.81 $(\mathrm{SD}=7.37)$ vs. $37.49(\mathrm{SD}=3.39)$ $\mathrm{t}=2.61 ; p=0.01)$ (Table 3$)$.

\section{Association with symptomatology ADHD symptomatology (ASRSv1.1)}

We constructed a model adding ASRSv1.1 score as predictor of the ERS, CERQ, and BES scores, analyzing only ADHD and ADHD+BPD patients. We found that the total ASRSv1.1 score was positively associated with ERS total score $(\beta=0.74, p<0.001)$ and all the subscales (Sensitivity: $\beta=0.38 ; p<0.001$; Arousal/Intensity: $\beta=$ 0.26; $p<0.001$; and Persistence: $\beta=0.13 ; p<0.001$ ). This association was also true when looking at attentional or hyperactive/impulsive symptoms (data not shown). 
Table 3 Comparisons of ERS, CERQ and BES results between ADHD and control samples

\begin{tabular}{|c|c|c|c|c|c|c|c|c|c|c|c|}
\hline & & ADHD & & Contro & & & & Control & & & \\
\hline & & $N=279$ & & $N=87^{2}$ & & $\mathrm{t}$ & $p$ & $N=258$ & & $\mathrm{t}$ & $p$ \\
\hline & & mean & sd & mean & sd & & & mean & $\mathrm{sd}$ & & \\
\hline ERS & Sensitivity & 22.31 & 8.78 & 16.29 & 8.61 & 5.61 & $<0.001$ & 16.43 & 8.37 & 7.93 & $<0.001$ \\
\hline & Arousal/Intensity & 15.84 & 7.06 & 13.11 & 6.3 & 3.22 & $<0.001$ & 11.48 & 6.33 & 7.51 & $<0.001$ \\
\hline & Persistence & 9.23 & 3.78 & 7.26 & 4.03 & 4.18 & $<0.001$ & 7.11 & 3.47 & 6.75 & $<0-001$ \\
\hline & Total & 47.38 & 18.39 & 36.66 & 17.52 & 4.79 & $<0.001$ & 35.02 & 17.14 & 8.03 & $<0.001$ \\
\hline & & $N=279$ & & $N=61$ & & $t$ & $p$ & $n=32^{d}$ & & $\mathrm{t}$ & $p$ \\
\hline & & mean & $\mathrm{sd}$ & mean & $\mathrm{sd}$ & & & mean & $\mathrm{sd}$ & & \\
\hline CERQ & Self-Blame & 11.53 & 3.75 & 8.22 & 2.96 & 14.19 & $<0.001$ & - & - & - & \\
\hline & Acceptance & 12.83 & 3.38 & 11.01 & 3.53 & 7.23 & $<0.001$ & 12.41 & 3.16 & 0.67 & 0.503 \\
\hline & Rumination & 12.94 & 3.86 & 10.46 & 3.72 & 9.12 & $<0.001$ & - & - & - & \\
\hline & Positive Refocusing & 9 & 3.68 & 10.01 & 3.53 & -3.91 & $<0.001$ & 9.5 & 3.81 & -0.73 & 0.469 \\
\hline & Refocusing on Planning & 12.94 & 3.63 & 13.03 & 3.89 & -0.33 & 0.744 & 13.84 & 3.5 & -1.33 & 0.183 \\
\hline & Positive Reapraisial & 12.45 & 3.89 & 12.46 & 4.07 & -0.03 & 0.972 & 14.56 & 3.9 & -2.91 & 0.003 \\
\hline & Putting into perspective & 12.27 & 3.82 & 11.64 & 3.91 & 2.24 & 0.01 & 11.44 & 2.55 & 1.19 & 0.232 \\
\hline & Catastrophizing & 8.66 & 3.6 & 6.05 & 2.43 & 12.68 & $<0.001$ & - & - & - & - \\
\hline & Blaming others & 8.84 & 3.53 & 6.38 & 2.69 & 11.43 & $<0.001$ & - & - & - & - \\
\hline & Adaptive Strategies Tot. & 59.49 & 13.29 & - & - & - & - & - & - & - & - \\
\hline & Non-adaptive Strategies Tot. & 41.97 & 10.66 & - & - & - & - & - & - & - & - \\
\hline & & $N=279$ & & $N=73^{6}$ & & $\mathrm{t}$ & $p$ & mean & sd & $\mathrm{t}$ & $p$ \\
\hline & & mean & sd & mean & $\mathrm{sd}$ & & & & & & \\
\hline BES & Cognitive & 32.39 & 3.86 & 37.62 & 3.46 & -10.52 & $<0.001$ & - & - & - & - \\
\hline & Affective & 39.81 & 7.37 & 37.49 & 3.39 & 2.61 & 0.01 & - & - & - & - \\
\hline & Total & 72.21 & 9.82 & 75.11 & 10.2 & -2.22 & 0.03 & - & - & - & - \\
\hline
\end{tabular}

Means, standard deviation (SD) and comparisons (coefficient and $p$ value) between ADHD and control samples derived from five independent studies for Emotion Regulation Scale (ERS), Cognitive Emotion Regulation Questionnaire (CERQ) and Basic Empathy Scale (BES)

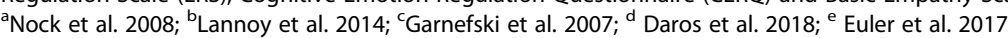

ASRSv1.1 total score was also significantly associated with higher score on non-adaptive cognitive strategies total score $(\beta=0.24, p<0.001)$ and with each of the non-adaptive subscales (data not shown). It was not associated with adaptive strategies $(\beta=0.03, p=0.641)$. The significant association was observed for attentional as well as hyperactive/impulsive symptoms.

ASRSv1.1 was significantly associated with higher empathy total score $(\beta=0.11, p<0.05)$, affective subscale score $(\beta=0.08, p<0.05)$, but not cognitive subscale score $(\beta=0.03, p=0.142)$. The association was found only with attentional symptoms $(\beta=0.32, p=0.001$ for empathy total score).

\section{$B D I-I I$ as a measure of current level of distress}

When adding BDI-II total score as predictor in the model, we found that current level of distress was associated with higher ERS total scores $(\beta=0.72, p<0.001)$. The difference between ADHD and BPD patients was no longer significant $(\beta=2.48, p=0.282)$, however the difference between ADHD and ADHD+BPD remained significant $(\beta=3.29 ; p<0.005)$.

BDI-II total score was also predictive of lower CERQ adaptive strategies $(\beta=-0.31, p<0.001)$ and higher non-adaptive strategies $(\beta=0.41, \quad p<0.001)$. When adjusting for BDI-II, the difference between groups was no longer significant $(\beta=-2.66, p=0.197$ and $\beta=-1.86$, $p=0.379$ for adaptive, and $\beta=-1.44, p=0.339$ and $\beta=$ $1.95, p=0.208$ for non-adaptive strategies, respectively for difference between ADHD and BPD and ADHD and ADHD+BPD).

BDI-II was not associated with empathy total score $(\beta=0,02, p=0.704)$.

\section{Association with $A D H D$ presentation}

ADHD patients with the combined presentation had higher ERS total scores $(\beta=6.72 ; p=0.002)$ than those with the attentional presentation. There were no differences between the two presentations of ADHD on CERQ adaptive and non-adaptive subscales $(\beta=1.64$; 
$p=0.318$ and $\beta=0.38 ; p=0.776)$ or on BES total score $(\beta=-0.87 ; p=0.452)$.

Comparing BPD patients either to patients with ADHD combined or only attentional presentations yielded similar results as comparing BPD patients to the entire ADHD patient group on the ERS total score, BES total score and CERQ adaptive and CERQ non-adaptive score.

\section{Taking into account the current depressive episode}

Thirty-three (47.13\%) BPD, 44 (15.77\%) ADHD and 25 (41.67\%) BPD + ADHD patients had a current major depressive episode. When adding current major depressive episode as a predictor in the model, we found that the difference between groups was still significant, with ADHD patients showing lower ERS total score than BPD patients $(\mathrm{b}=-8.79 ; p=0.001)$ and ADHD+BPD patients $(\beta=-13.33 ; p<0.001)$. This was true for all the ERS subscales.

Current major depressive episode was also associated with lower CERQ adaptive strategies $(\beta=-5.27 ; p=0.004)$ and with higher CERQ non-adaptive strategies $(\beta=4.63$; $p=0.001$ ). When adjusting for current major depressive episode, ADHD patients still showed higher CERQ adaptive strategies than BPD patients $(\beta=4.78 ; p=0.021)$, but the difference with ADHD+BPD was no longer significant $(\beta=$ 3.92; $p=0.064$ ). Furthermore, with this adjustment the difference between ADHD and BPD for CERQ non-adaptive strategies was no longer significant $(\beta=-2.87 ; p=0.074)$, but was still significant for the comparison between ADHD and ADHD+BPD $(\beta=-561 ; p=0.001)$ with lower scores in ADHD patients.

No association between BDI-II and empathy total score was found $(\beta=1.67 ; p=0.126)$.

\section{Discussion}

We found that ADHD patients, although having more ED than community-based psychiatric patients or controls, had significantly better emotion regulation and more efficient emotion regulation strategies than subjects suffering from BPD, and BPD and ADHD combined. Our results indeed showed lower emotional reactivity, better use of adaptive cognitive strategies and lesser use of non-adaptive strategies in ADHD patients than in subjects of the two other groups. On the other hand, cognitive and affective empathy abilities were similar between groups. Finally, emotion regulation difficulties were associated with ADHD symptomatology (specifically intensity, persistence and reactivity, and use of non-adaptive strategies).

We found that patients with ADHD had higher scores on scales measuring ED compared to community-based psychiatric patients. Previous studies suggested a slow return to emotional baseline, increased intensity and instability of negative emotions, and a predominance of negative emotions in ADHD [3, 4, 10]. Our findings thus add to the current body of literature suggesting difficulties in regulating emotions in ADHD [24, 49]. These findings may be related to the fact that ADHD patients showed an overall higher use of non-adaptive cognitive emotion regulation strategies than healthy controls. They indeed showed similar scores on the CERQ nonadaptive 'blaming others' and 'rumination' subscales as BPD patients. Rumination was previously found to be overused by BPD patients and linked to the persistence of negative emotions $[19,50]$. Our results thus suggest that, similar to $\mathrm{BPD}$, poor cognitive emotion regulation strategies such as 'rumination' as well as others such as 'self-blame', 'blaming others' and 'catastrophizing' to a lesser extent, play a role in ED in ADHD [17]. Of note, our results also suggest that ADHD patients mainly differ from controls by a higher tendency to use nonadaptive cognitive strategies rather than adaptive ones, similar to BPD patients $[18,51]$. Nonetheless, ADHD patients still had overall less emotion sensitivity, less emotion arousal and intensity, and less persistence of the emotion than BPD and BPD + ADHD patients. Compared to the other two patient groups, they also used more adaptive and less non-adaptive cognitive emotional regulation strategies. These results suggest that, although of importance in ADHD, emotion dysregulation is not as central as it is in BPD and may explain only a part of the difficulties found in this disorder. Nevertheless, severity of ADHD was associated with higher emotional reactivity and with higher use of non-adaptive cognitive strategies. This is in agreement with other evidence suggesting that ADHD severity is closely related to difficulties in emotion regulation $[7,8]$. This is also concordant with findings in BPD, which link more use of maladaptive cognitive emotion regulation strategies with higher rates of potentially harmful behaviours and severity of the disorder $[17,18]$. Thus one might think that, as in BPD, using maladaptive cognitive emotion regulation strategies (which have more short-term benefits) more often than adaptive strategies helps maintain ED, which secondarily leads to increased severity of ADHD attentional and hyperactive-impulsive symptoms [17, 20, 22, 52]. These observations stress the impact of emotional symptoms on ADHD prognosis and the value of early diagnosis to address them, moreover considering the evidence that ED symptoms could mediate the relationship between childhood ADHD and adulthood BPD symptoms [13]. In addition, knowing that ED has been associated with poor global functioning, poor prognosis, severity of ADHD, higher rate of comorbidities and persistence of ADHD in adulthood, early interventions targeting this dimension are crucially needed $[1,4,7-9$, 53]. The importance of a long-term integrative approach can be highlighted by the limited effect of medication on 
ED [4]. Indeed research has shown that while pharmacotherapy can improve to a certain extent these symptoms, its effect seems to be lesser than on the other wellknown "dysexecutive" ADHD symptoms [4, 5, 54].

We found that ADHD as well as BPD displayed lower empathy than a sample of adolescents not suffering from psychiatric disorder. This slight deficit in empathy might contribute, as in BPD, to poorer emotion regulation capacities $[14,55]$. Previous research has indeed found that ADHD patients have empathic capacities that are slightly below those of healthy controls. These poorer capacities are related to difficulties in perceiving contextual information possibly linked to deficits in directing attention on emotional signals, to deficits in reflexive functioning, and to deficits in emotional face recognition $[9,14,23]$. Thus targeting empathy, either directly or indirectly, in ADHD during treatment might help decrease ED and subsequently the severity of the disorder [56, 57]. Indeed, improving mentalizing capacities - defined as linking one's own and other people's actions to mental states which are thus closely related to empathy - is promising for improving emotion regulation in adults suffering from ADHD [57].

Concerning ADHD presentation, the combined type was associated with higher emotional reactivity as previously shown in other studies [2,4]. No difference in the use of cognitive emotional regulation strategies was observed.

The hypothesis of a possible additive effect of both disorders (ADHD + BPD) was not really corroborated by our results since no significant difference between BPD and ADHD+BDP was found. However, this observation might be due to a ceiling effect as the scales we used were designed to assess emotions present in the general population and not in highly emotionally dysregulated samples such as BPD.

Part of the differences between groups was related to the level of distress as measured by the BDI-II [32]. Our results showed a high level of correlation between current distress, emotional reactivity and the use of poor cognitive emotion regulation strategies in ADHD but also in BPD. This result highlights the substantial negative impact of ED on patients' well-being and quality of life $[4,9]$. It is essential to point out the low percentage of comorbidities compared to previous research, which could be explained by our study focus being mainly on current comorbidities and not on their lifetime occurrence. They were also assessed clinically without further investigations.

Our study has several limitations. Firstly, we did not have a control population and used BPD patients as a comparison to assess ED, cognitive emotion regulation strategies and empathy in ADHD patients. We nevertheless compared our patients to samples from the general population, although not matched on age and gender, derived from others studies and we are quite confident that the assessment of emotion components in our study is a true reflection of the difficulties endured by patients suffering from BPD and ADHD. Secondarily, there was a substantial difference in the size of our three groups, the ADHD group being the largest one. This may have slightly biased our results keeping in mind that ED in ADHD subjects was the main focus of our study, the two other groups being here considered as comparison samples. Thirdly we used self-report measures to assess the different aspects of emotions. However it has previously been shown that self-report measures are reliable if used in combination with interviews, which was the case in our study [58]. Finally, ED in our sample might be better explained by current comorbid disorders, especially current major depressive episode. However, when we adjusted for current major depressive episode, ADHD was still associated with less ED and more use of adaptive cognitive strategies than BPD. Therefore the difference in ED reflected by the ERS couldn't be totally explained by the presence of more clinical depression.

\section{Conclusions}

Our findings support the importance of exploring facets of ED as well as cognitive emotion regulation strategies in ADHD. These may, as in BPD, be an important feature of the disorder and be associated with its severity. In general, our results showed that maladaptive cognitive emotion strategies for management of emotion, such as 'self-blame', 'catastrophizing,' 'other-blame' and 'rumination' are often used by ADHD patients and should be considered in individual and group psychotherapeutic approaches offered to these patients. Furthermore, ADHD patients showed a better use of cognitive emotional regulation strategies and less emotional reactivity than BPD patients. Similarities were also found between these populations, such as the tendency of using 'blaming others' and the absence of a difference in cognitive empathy, possibly suggesting a common deficit in perception of self and others in emotionally triggering situations.

\section{Abbreviations \\ ADHD: Attention deficit hyperactivity disorder; ARSV-v1.1: Adult ADHD Self- Report Scale; BDI-II: Beck Depression Inventory II.; BES-A: Basic Empathy Scale; BPD: Borderline personality disorder; BSL-23: Borderline Symptoms Check-List; CERQ: Cognitive Emotional Regulation Questionnaire; DIVA 2.0: Diagnostic Interview for ADHD in adults; ED: Emotional dysregulation; ERS: Emotion Reactivity Scale; SCID-II: Screening Interview for Axis II disorders; SD: Standard deviation}

\section{Acknowledgments}

We would like to thank Julia Griem for her editorial support.

\section{Authors' contributions}

ER contributed in the conception, the design, the acquisition, the analysis the interpretation of data of the study and the drafting of the manuscript. SE contributed in the interpretation of data and the drafting of the manuscript. PP,

$\mathrm{RN}, \mathrm{KD}, \mathrm{RH}$ and EP contributed in the acquisition of data. NP and SW participated 
in the conception, the design, the interpretation of data of the study and the drafting of the manuscript. All authors read and approved the final manuscript.

\section{Funding}

The study was supported by the Swiss National Center of Competence in Research, "Synapsy: the Synaptic Basis of Mental Diseases" [grant number. 51NF40-185897].

\section{Availability of data and materials}

The datasets used and/or analyzed during the current study are available from the corresponding author on reasonable request.

\section{Ethics approval and consent to participate}

The study was approved by the ethics committee of University Hospitals of Geneva and all subjects provided informed written consent.

\section{Consent for publication}

Not applicable.

\section{Competing interests}

The authors declare that they have no competing interests.

\section{Author details}

${ }^{1}$ TRE Unit, Department of Psychiatry, Division of Psychiatric Specialties, Department of Mental Health and Psychiatry, University Hospitals of Geneva, 20bis rue de Lausanne, Geneva, Switzerland. ${ }^{2}$ Department of Consultation Psychiatry and Psychosomatics, University Hospital Zurich and University of Zurich, Zurich, Switzerland. ${ }^{3}$ Department of Psychiatry, Faculty of Medicine, University of Geneva, Geneva, Switzerland. ${ }^{4}$ Pôle de Psychiatrie, Santé Mentale et Addictologie, University Hospital Strasbourg, Strasbourg, France. ${ }^{5}$ INSERM 1114, Strasbourg, France. ${ }^{6}$ FMTS, University of Strasbourg, Strasbourg, France.

Received: 26 February 2019 Accepted: 25 June 2019

Published online: 18 July 2019

\section{References}

1. Morstedt B, Corbisiero S, Bitto H, Stieglitz RD. Emotional symptoms and their contribution to functional impairment in adults with attention-deficit/ hyperactivity disorder. Atten Defic Hyperact Disord. 2016;8(1):21-33.

2. Vidal R, Valero S, Nogueira M, Palomar G, Corrales M, Richarte V, et al. Emotional lability: the discriminative value in the diagnosis of attention deficit/hyperactivity disorder in adults. Compr Psychiatry. 2014;55(7):1712-9.

3. Hirsch O, Chavanon M, Riechmann E, Christiansen H. Emotional dysregulation is a primary symptom in adult attention-deficit/hyperactivity disorder (ADHD). J Affect Disord. 2018;232:41-7.

4. Faraone SV, Rostain AL, Blader J, Busch B, Childress AC, Connor DF, et al. Practitioner review: emotional dysregulation in attention-deficit/ hyperactivity disorder - implications for clinical recognition and intervention. J Child Psychol Psychiatry. 2019;60(2):133-50.

5. Posner J, Kass E, Hulvershorn L. Using stimulants to treat ADHD-related emotional lability. Curr Psychiatry Rep. 2014;16(10):478.

6. Qian Y, Chang W, He X, Yang L, Liu L, Ma Q, et al. Emotional dysregulation of ADHD in childhood predicts poor early-adulthood outcomes: a prospective follow up study. Res Dev Disabil. 2016;59:428-36.

7. Banaschewski T, Jennen-Steinmetz C, Brandeis D, Buitelaar JK, Kuntsi J, Poustka L, et al. Neuropsychological correlates of emotional lability in children with ADHD. J Child Psychol Psychiatry. 2012;53(11):1139-48.

8. Sobanski E, Banaschewski T, Asherson P, Buitelaar J, Chen W, Franke B, et al. Emotional lability in children and adolescents with attention deficit/ hyperactivity disorder (ADHD): clinical correlates and familial prevalence. J Child Psychol Psychiatry. 2010;51(8):915-23.

9. Shaw P, Stringaris A, Nigg J, Leibenluft E. Emotion dysregulation in attention deficit hyperactivity disorder. Am J Psychiatry. 2014;171(3):276-93.

10. Skirrow C, Ebner-Priemer U, Reinhard I, Malliaris Y, Kuntsi J, Asherson P. Everyday emotional experience of adults with attention deficit hyperactivity disorder: evidence for reactive and endogenous emotional lability. Psychol Med. 2014:44(16):3571-83.

11. Matthies SD, Philipsen A. Common ground in attention deficit hyperactivity disorder (ADHD) and borderline personality disorder (BPD)-review of recent findings. Borderline Personal Disord Emot Dysregul. 2014;1:3.
12. Philipsen A. Differential diagnosis and comorbidity of attention-deficit/ hyperactivity disorder (ADHD) and borderline personality disorder (BPD) in adults. Eur Arch Psychiatry Clin Neurosci. 2006;256(Suppl 1):i42-6.

13. Fossati A, Gratz KL, Borroni S, Maffei C, Somma A, Carlotta D. The relationship between childhood history of ADHD symptoms and DSM-IV borderline personality disorder features among personality disordered outpatients: the moderating role of gender and the mediating roles of emotion dysregulation and impulsivity. Compr Psychiatry. 2015;56:121-7.

14. Perroud N, Badoud D, Weibel S, Nicastro R, Hasler R, Kung AL, et al. Mentalization in adults with attention deficit hyperactivity disorder: comparison with controls and patients with borderline personality disorder. Psychiatry Res. 2017;256:334-41.

15. Cackowski S, Krause-Utz A, Van Eijk J, Klohr K, Daffner S, Sobanski E, et al. Anger and aggression in borderline personality disorder and attention deficit hyperactivity disorder - does stress matter? Borderline Personal Disord Emot Dysregul. 2017:4:6.

16. Cavelti M, Corbisiero S, Bitto H, Moerstedt B, Newark P, Faschina S, et al. A comparison of self-reported emotional regulation skills in adults with attention-deficit/hyperactivity disorder and borderline personality disorder. J Atten Disord. 2017:1087054717698814. https://doi.org/10.1177/ 1087054717698814

17. Carpenter RW, Trull TJ. Components of emotion dysregulation in borderline personality disorder: a review. Curr Psychiatry Rep. 2013;15(1):335.

18. Daros AR, Guevara MA, Uliaszek AA, McMain SF, Ruocco AC. Cognitive emotion regulation strategies in borderline personality disorder: diagnostic comparisons and associations with potentially harmful behaviors Psychopathology. 2018;51(2):83-95.

19. Baer RA, Peters JR, Eisenlohr-Moul TA, Geiger PJ, Sauer SE. Emotion-related cognitive processes in borderline personality disorder: a review of the empirical literature. Clin Psychol Rev. 2012;32(5):359-69.

20. Selby EA, Anestis MD, Bender TW, Joiner TE Jr. An exploration of the emotional cascade model in borderline personality disorder. J Abnorm Psychol. 2009;118(2):375-87.

21. Cheavens JS, Zachary Rosenthal M, Daughters SB, Nowak J, Kosson D, Lynch TR, et al. An analogue investigation of the relationships among perceived parental criticism, negative affect, and borderline personality disorder features: the role of thought suppression. Behav Res Ther. 2005;43(2):257-68.

22. Rosenthal MZ, Cheavens JS, Lejuez CW, Lynch TR. Thought suppression mediates the relationship between negative affect and borderline personality disorder symptoms. Behav Res Ther. 2005;43(9):1173-85.

23. Borhani $K$, Nejati $V$. Emotional face recognition in individuals withattention-deficit/hyperactivity disorder: a review article. Dev Neuropsychol. 2018;43(3):256-77.

24. Bunford N, Evans SW, Wymbs F. ADHD and emotion dysregulation among children and adolescents. Clin Child Fam Psychol Rev. 2015;18(3):185-217.

25. Jusyte A, Gulewitsch MD, Schonenberg M. Recognition of peer emotions in children with ADHD: evidence from an animated facial expressions task. Psychiatry Res. 2017;258:351-7.

26. Bunford N, Evans SW, Becker SP, Langberg JM. Attention-deficit/ hyperactivity disorder and social skills in youth: a moderated mediation model of emotion dysregulation and depression. J Abnorm Child Psychol. 2015:43(2):283-96.

27. Bisch J, Kreifelts B, Bretscher J, Wildgruber D, Fallgatter A, Ethofer T. Emotion perception in adult attention-deficit hyperactivity disorder. J Neural Transm (Vienna). 2016;123(8):961-70.

28. Runions K, Rao P, Wong JWY, Zepf FD. Attention-deficit/hyperactivity disorder and the encoding of emotional information. Acta Psychiatr Scand. 2017;135(6):503-5.

29. Sullivan EL, Holton KF, Nousen EK, Barling AN, Sullivan CA, Propper CB, et al. Early identification of ADHD risk via infant temperament and emotion regulation: a pilot study. J Child Psychol Psychiatry. 2015;56(9):949-57.

30. Coutinho TV, Reis SPS, da Silva AG, Miranda DM, Malloy-Diniz LF. Deficits in response inhibition in patients with attention-deficit/hyperactivity disorder: the impaired self-protection system hypothesis. Front Psych. 2017:8:299.

31. Schneidt A, Jusyte A, Schonenberg M. Interpretation of ambiguous facial affect in adults with attention-deficit/hyperactivity disorder. Eur Arch Psychiatry Clin Neurosci. 2018.

32. Shushakova A, Ohrmann P, Pedersen A. Exploring deficient emotion regulation in adult ADHD: electrophysiological evidence. Eur Arch Psychiatry Clin Neurosci. 2018;268(4):359-71. 
33. Caci HM, Morin AJ, Tran A. Prevalence and correlates of attention deficit hyperactivity disorder in adults from a French community sample. J Nerv Ment Dis. 2014;202(4):324-32.

34. Nicastro R, Prada P, Kung AL, Salamin V, Dayer A, Aubry JM, et al. Psychometric properties of the French borderline symptom list, short form (BSL-23). Borderline Personal Disord Emot Dysregul. 2016;3:4.

35. Ramos-Quiroga JA, Nasillo V, Richarte V, Corrales M, Palma F, Ibanez P, et al. Criteria and concurrent validity of DIVA 2.0: a semi-structured diagnostic interview for adult ADHD. J Atten Disord. 2016

36. First M, Gibbon M. The structured clinical interview for DSM-IV Axis I disorders (SCID-I) and the structured clinical interview for DSM-IV Axis II disorders (SCID-II). In: Hilsenroth M, Segal D, editors. Comprehensive handbook of psychological assessment, Vol 2: personality assessment. Hoboken: Wiley; 2004. p. 134-43.

37. Beck A, Steer R, Brown G. BDI-II, Beck depression inventory: manual psychological corporation; 1996.

38. Nicastro R, Desseilles M, Prada P, Weibel S, Perroud N, Gex-Fabry M. Subjective distress associated with adult ADHD: evaluation of a new selfreport. Atten Defic Hyperact Disord. 2018;10(1):77-86.

39. Nock MK, Wedig MM, Holmberg EB, Hooley JM. The emotion reactivity scale: development, evaluation, and relation to self-injurious thoughts and behaviors. Behav Ther. 2008;39(2):107-16.

40. Lannoy S, Heeren A, Rochat L, Rossignol M, Van der Linden M, Billieux J. Is there an all-embracing construct of emotion reactivity? Adaptation and validation of the emotion reactivity scale among a French-speaking community sample. Compr Psychiatry. 2014;55(8):1960-7.

41. Claes $L$, Smits $D$, Bijttebier $P$. The Dutch version of the emotion reactivity scale: validation and relation with various behaviors in a sample of high school students. Eur J Psychol Assess. 2013;1:1-7.

42. Garnefski N, Kraaij V, Spinhoven P. Negative life events, cognitive emotion regulation and emotional problems. Personal Individ Differ. 2001;30:1311-27.

43. Jermann F, Van der Linden M, d'Acremont M, Zermatten A. Cognitive emotion regulation questionnaire (CERQ): confirmatory factor analysis and psychometric properties of the French translation. Eur J Psychol Assess. 2006:22:126-31.

44. Jolliffe D, Farrington DP. Development and validation of the basic empathy scale. J Adolesc. 2006:29(4):589-611.

45. Carre A, Stefaniak N, D'Ambrosio F, Bensalah L, Besche-Richard C. The basic empathy scale in adults (BES-A): factor structure of a revised form. Psychol Assess. 2013;25(3):679-91.

46. Bensalah L, Stefaniak N, Carre A, Besche-Richard C. The basic empathy scale adapted to French middle childhood: structure and development of empathy. Behav Res Methods. 2016:48(4):1410-20.

47. Garnefski N, Kraaij V. The cognitive emotion regulation questionnaire psychometric features and prospective relationships with depression and anxiety in adults. Eur J Psychol Assess. 2007;23:141-9.

48. Euler F, Steinlin C, Stadler C. Distinct profiles of reactive and proactive aggression in adolescents: associations with cognitive and affective empathy. Child Adolesc Psychiatry Ment Health. 2017;11:1.

49. Nigg JT, Silk KR, Stavro G, Miller T. Disinhibition and borderline personality disorder. Dev Psychopathol. 2005;17(4):1129-49.

50. Baer RA, Sauer SE. Relationships between depressive rumination, anger rumination, and borderline personality features. Personal Disord. 2011;2(2): 142-50.

51. Lotfi M, Amini M, Fathi A, Karami A, Ghiasi S, Sadeghi S. Cognitive emotion regulation strategies in prisoners with borderline personality disorder. J Pract Clin Psychol. 2018;6(3):153-8.

52. Kim S, Sharp C, Carbone C. The protective role of attachment security for adolescent borderline personality disorder features via enhanced positive emotion regulation strategies. Personal Disord. 2014:5(2):125-36.

53. Christiansen H, Hirsch O, Albrecht B, Chavanon ML. Attention-deficit/ hyperactivity disorder (ADHD) and emotion regulation over the life span. Curr Psychiatry Rep. 2019;21(3):17.

54. Catala-Lopez F, Hutton B, Nunez-Beltran A, Page MJ, Ridao M, Macias SaintGerons D, et al. The pharmacological and non-pharmacological treatment of attention deficit hyperactivity disorder in children and adolescents: a systematic review with network meta-analyses of randomised trials. PLoS One. 2017;12(7):e0180355.

55. Mancke F, Herpertz SC, Bertsch K. Aggression in borderline personality disorder: a multidimensional model. Personal Disord. 2015;6(3):278-91.
56. Jones B, Allison E. An integrated theory for attention-deficit hyperactivity disorder [ADHD]; 2010. p. 279-95.

57. Badoud D, Rüfenacht E, Debbané M, Perroud N. Mentalization-based treatment for adults with attention-deficit/hyperactivity disorder: a pilot study; 2018

58. Silverstein MJ, Faraone SV, Alperin S, Biederman J, Spencer TJ, Adler LA. How informative are self-reported adult attention-deficit/hyperactivity disorder symptoms? An examination of the agreement between the adult attention-deficit/hyperactivity disorder self-report scale V1.1 and adult attention-deficit/hyperactivity disorder investigator symptom rating scale. J Child Adolesc Psychopharmacol. 2018;28(5):339-49.

\section{Publisher's Note}

Springer Nature remains neutral with regard to jurisdictional claims in published maps and institutional affiliations.

\section{Ready to submit your research? Choose BMC and benefit from:}

- fast, convenient online submission

- thorough peer review by experienced researchers in your field

- rapid publication on acceptance

- support for research data, including large and complex data types

- gold Open Access which fosters wider collaboration and increased citations

- maximum visibility for your research: over $100 \mathrm{M}$ website views per year

At BMC, research is always in progress.

Learn more biomedcentral.com/submissions 